\title{
Monte Carlo simulation of the glass transition in polymer melts: extended mode-coupling analysis
}

\author{
Jörg Baschnagelł $\S$ and Matthias Fuchs $\ddagger$ \\ † Institut für Physik, Johannes-Gutenberg Universität, Staudinger Weg 7, D-55099 Mainz, \\ Germany \\ ‡ Physik Department, Technische Universität Mïnchen, D-85748 Garching, Germany
}

Received 27 April 1995

\begin{abstract}
In this paper, we reanalyse the incoherent intermediate scattering function, determined by a Monte Carlo simulation of a polymer melt, in the framework of the extended mode-coupling theory (MCT). A previous analysis with the idealized MCT showed systematic deviations between theory and simulation at low temperatures, which could be qualitatively attributed to the neglect of hopping processes. If these hopping processes are taken into account quantitatively by the application of the extended MCT, the discrepancies at low temperanres disappear, and a (quite) accurate estimate of the critical temperature becomes possible.
\end{abstract}

\section{Introduction}

The liquid-glass transition is preceded by a strong increase of the structural relaxation time. Typically, a liquid in the vicinity of the triple point relaxes on the time scale of $10^{-12}$ s. When the liquid is progressively supercooled, this time scale first rises gradually up to about $10^{-9} \mathrm{~s}$, and then very steeply over many orders of magnitude. The liquid vitrifies as soon as the relaxation time adopts macroscopic values (i.e. $10^{3} \mathrm{~s}$ or larger) $[1,2,3,4]$. A comparable dependence of the structural relaxation time on temperature is not observed during the crystallization process and may thus be looked upon as a generic feature of the glass transition.

An attempt to explain this dynamical behaviour of supercooled liquids was made by the mode-coupling approach to the structural glass transition (see the recent reviews $[3,4,5,6]$ ). Mode-coupling theory (MCT) predicts that there is a critical temperature $T_{c}$, situated well above the calorimetric glass transition temperature, at which the dynamics of the supercooled liquid changes qualitatively. This change in the dynamics results from the competition between an ergodicity breaking process, the cage effect (i.e. rattling motion of the particles inside the cages, dynamical changes of the cage configurations) and an ergodicity restoring process, which is identified with thermally activated hopping processes $[7,8]$ following a conjecture of Goldstein [9]. The consequences of the cage effect for the dynamics of the supercooled liquid are treated in the idealized version of the MCT $[3,4,5,6]$, whereas the physical origin of the hopping processes as well as the resulting corrections to the idealized theory are the subject of the extended MCT $[8,10,11,12]$.

The primary result of the extended MCT is the prediction of a (rather) universal dynamical scaling law in an intermediate frequency (time) range. Irrespective of the exact

\$ To whom correspondence should be addressed. 
microscopic nature of the ergodicity restoring process, of its wave vector and (to a large extent) of its frequency dependence, it was found that a single relevant control parameter suffices to describe the change of the dynamics [8]. Although no microscopic theory for thermally activated processes in supercooled liquids has yet been worked out, the extended MCT makes precise statements which allow direct experimental tests of the theory [12]. The extended MCT was applied to interpret the light scattering spectra of the ionic salt calcium potassium nitrate $(\mathrm{CKN})$, the molecular glass former salol [13] and propylene carbonate (PC) [14]. Originally, the spectra for CKN and salol were analysed with the idealized theory in a temperature interval ranging from about the melting point to the calorimetric glass transition temperature $[15,16,17,18,19]$. This light scattering analysis found that the studied temperature interval can be divided into two parts. For temperatures sufficiently above $T_{c}$, the idealized theory correctly describes the spectra in the whole frequency range between the band of the microscopic excitations and the final $\alpha$-relaxation (i.e. over about two to three decades around the minimum of the spectrum). Its applicability is limited to the high-frequency wing of this spectral window if the temperature is in the vicinity of or below $T_{c}$. In this low-temperature region the experimental data at smaller frequencies lie systematically above the theoretical predictions, indicating that both $\mathrm{CKN}$ and salol tend to relax more strongly than the idealized MCT predicts $[13,14]$.

For polymer melts, a similar observation was made in a Monte Carlo simulation of a coarse-grained lattice model [20]. In this simulation the incoherent intermediate scattering function $\phi_{q}^{s}(t)$ was calculated. This function exhibits a two-step decay in a certain temperature interval, which is the typical relaxation behaviour predicted by MCT for $\phi_{g}^{s}(t)$ close to $T_{c}[3,4,5,6]$. Therefore the time evolution of $\phi_{q}^{s}(t)$ was quantitatively analysed by the idealized MCT, leading to similar results as found in the light scattering experiments mentioned above. There is a high-temperature regime, where the idealizcd theory accounts for the decay of $\phi_{4}^{s}(t)$ over about three decades in time, and a low-temperature regime, where it overestimates the freezing tendency of the melt. Since this clearly indicates the presence of a further relaxation process which should be captured by the extended MCT, we decided to check whether the inclusion of hopping processes is able to resolve the discovered discrepancies between the idealized theory and the simulation data.

This paper summarizes the results of this analysis which represents the first quantitative application of extended MCT in the time domain. It is structured in the following way: section 2 reviews some aspects of the model and of the simulation. Section 3 provides the necessary theoretical background for the fits. Section 4 summarizes and discusses the results of the extended mode-coupling analysis, and section 5 presents our conclusions.

\section{Aspects of the model and the simulation}

The polymer melt is simulated with the bond fluctuation model [21] which is a coarsegrained lattice model [22]. A monomer of such a coarse-grained model does not directly correspond to a chemical monomer, but rather to a group of chemical monomers which may be represented by an effective bead in their centre of gravity (see figure 1). For simple polymers, such as polyethylene, this group roughly contains three to five monomers [23]. Since the polymers of the bond fluctuation model have a length $N=10$ in this simulation, they represent rather short polyethylene chains having a size that is much smaller than the entanglement length $[24,25]$. Therefore entanglements cannot be responsible for the dynamic features to be discussed below.

This coarse graining has two consequences. First, the effective bond vectors, resulting from the coarse-graining procedure along the chemical polymers, are able to fluctuate in 

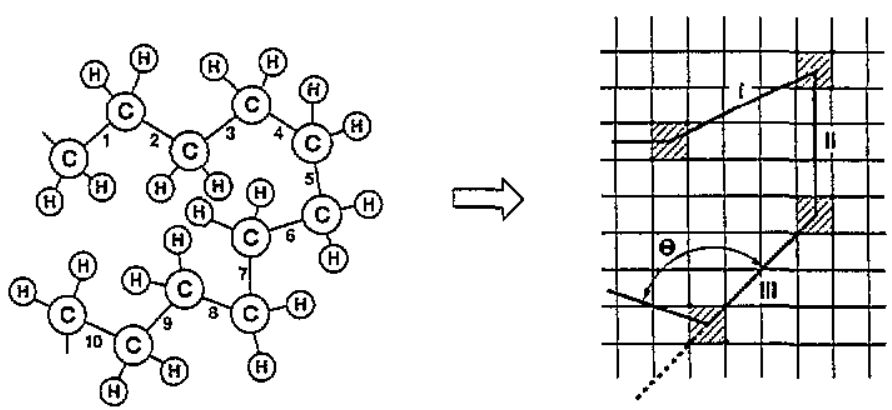

Figure 1. Schematic illustration of the construction of a coarse-grained model for a macromolecule such as polyethylene. In the example shown here, the subchain formed by the three $\mathrm{C}-\mathrm{C}$ bonds labelled $1,2,3$ is represented by the effective bond labelled as $\mathbf{I}$, the subchain formed by the three bonds $4,5,6$ is represented by the effective bond labelled as $\mathbf{~}$, etc. In the bond fluctuation model on the square (or simple cubic) lattice the length $b$ of the effective bond is allowed to fluctuate in a certain range $b_{\min } \leqslant b \leqslant b_{\max }$, and excluded-volume interactions are modelled by assuming that each bond occupies a plaquette (or cube) of four (eight) neighbouring lattice sites, which then are all blocked for further occupation.

both length and direction to a much larger extent than their chemical counterparts which are kept close to the equilibrium values by stiff potentials $[26,27,28]$. The bond fluctuation model maps this flexibility of the bond vectors onto a lattice by associating a monomer with a whole unit cell on a simple cubic lattice [21]. The monomers interact via a hard-core potential with each other. In addition to this excluded volume interaction an energy function $\mathcal{H}(b)$ is associated with each bond vector $b$, which favours the bonds of length $b=3$ and direction along the lattice axes (i.e. $\mathcal{H}(b)=0$ ) in comparison to all other available bond vectors (i.e. $\mathcal{H}(b)=\epsilon$ ) [29]. During the simulation a monomer is selected at random, and a move is attempted over the distance of one lattice spacing in a randomly chosen direction. If the adjacent lattice sites in the chosen direction are empty (excluded volume restriction) and if the resulting bond vectors belong to the set of allowed bonds [21] (restriction of chain connectivity), the move is accepted with probability $\exp \left[-\Delta \mathcal{H} / k_{\mathrm{B}} T\right]$, where $\Delta \mathcal{H}$ is the change in energy connected with the attempted jump. In this way temperature is introduced in the simulation [30]. When the temperature decreases, not only the bonds, but also the chains tend to stretch out under the influence of the above-described energy function [29]. This energy function thus leads, on the one hand, to a stiffening of the chains with decreasing temperature, an effect which is also observed in many interesting attempts to model the glass transition of polyethylene rather realistically $[26,27,28]$, and on the other hand it generates a strong competition between the energetic and packing constraints of the chains [29]. This competition drastically slows down the structural relaxation of the melt at low temperatures if the density $\phi$ is large enough [29]. We chose $\phi=0.5 \overline{3}$, resulting from $\phi=8 N P / L^{3}$ with the number of polymers per simulation box $P=180$ and the linear dimension of the simulation box $L=30$ (i.e. a volume fraction of $53 . \overline{3} \%$ of the lattice sites is occupied by monomers) [29]. The simulation box thus contains 1800 monomers. To improve the statistics, 16 independent configurations of this system were run in paralleI so that the total statistics of the data to be presented involves 28800 monomers.

The second consequence of the coarse graining is that the motion of the effective bead essentially becomes stochastic and thus comparable to the Monte Carlo dynamics. If one uses again polyethylene as an example, a jump of a monomer on the lattice may be related to the change of the conformation of a subunit comprising three to five monomers, i.e. 
to a change of the torsional angle [31]. Since the relaxation time of such a change in an equilibrium polyethylene melt is of the order of $10^{-11} s[26,32]$ and the acceptance rate of a jump in the bond fluctuation model is about $10^{-1}$ at the considered density [33], the intrinsic time unit of the simulation, the Monte Carlo step (MCS), should approximately correspond to a picosecond. A similar identification between the Monte Carlo and the physical time unit was obtained in a different application of the bond fluctuation model, in which the model potential was tailored in such a way that bisphenol-A-polycarbonate could be simulated $[34,35]$. Therefore the estimate $1 \mathrm{MCS} \approx 10^{-12} \mathrm{~s}$ suggests that a Monte Carlo simulation over six to seven decades in time describes dynamical processes ranging from picoseconds to $10^{-5} \mathrm{~s}$. Certainly, the description of the dynamics by the bond fluctuation model on the picosecond time scale is unrealistic. The bond fluctuation model cannot reliably mimic dynamical processes on such short time scales. Processes on this time scale are caused by phonons which are absent from the model, since the monomers are moved in discrete steps of one lattice constant in the simulation. However, this loss of the phonon contribution does not exclude a comparison with the MCT because the MCT attempts to describe the dynamics of a supercooled liquid on time scales which are larger than the microscopic excitation band $[3,4,5,6]$. For a significant test of the MCT the neglect of phonons in the simulation is thus not a drawback. It is more important to have long simulation times and to obtain good statistics. Good statistics is necessary for a quantitative comparison with the $\mathrm{MCT}$, whereas long simulation times are required to sample the time interval pertinent to MCT effects sufficiently and to realize the theoretical precondition of thermal equilibrium in the important temperature region as much as possible.

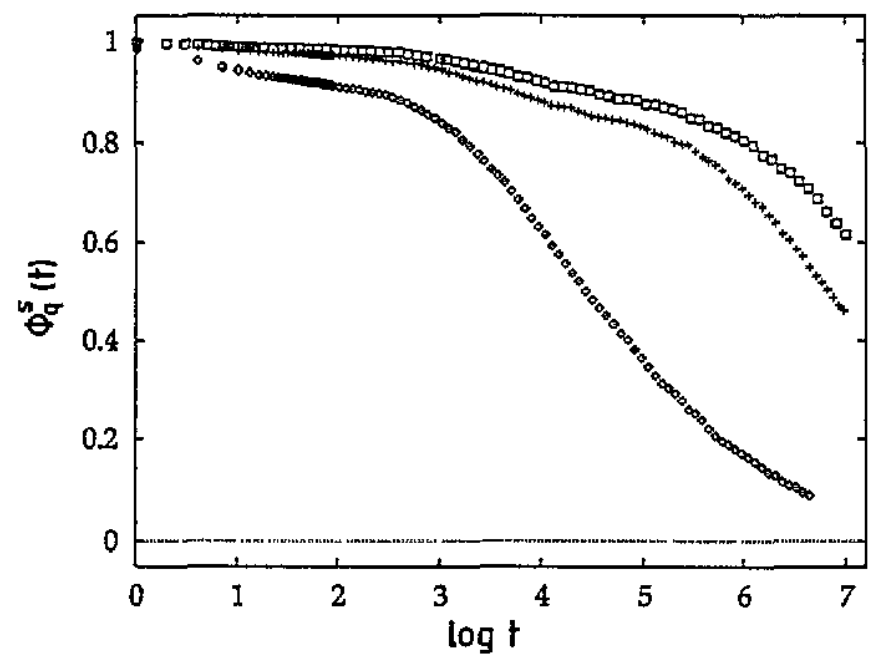

Figure 2. Comparison of the time dependence of $\phi_{q}^{s}(t)$ at the external temperature $T=0.16$ after the melt has been equilibrated for $1.4 \times 10^{7} \mathrm{MCS}(\square), 4 \times 10^{6} \mathrm{MCS}(+)$ and $6 \times 10^{3} \mathrm{MCS}$ $(\diamond)$. Note that the curve with the smallest equilibration time has decayed much more than the others and that a two-step process is not visible. This two-step process is observed for the data equilibrated for $4 \times 10^{6} \mathrm{MCS}$ and remains present if the equilibration time is further increased.

How an insufficient equilibration time may affect the dynamical behaviour of $\phi_{q}^{s}(t)$ is exemplified in figure 2. This figure compares the relaxation behaviour of $\phi_{q}^{s}(t)$ recorded after three different equilibration times, i.e. after $6 \times 10^{3} \mathrm{MCS}(\diamond), 4 \times 10^{6} \mathrm{MCS}(+)$ and $1.4 \times 10^{7} \mathrm{MCS}(\square)$. All three simulations were done at a temperature of $T=0.16$ and at 
the maximum of the static structure factor $q=2.92$ [30]. The curves for the two smaller equilibration times are reproduced from [20]. The one corresponding to an equilibration period of $4 \times 10^{6} \mathrm{MCS}$ is for the data used in the idealized mode-coupling analysis [20], and for which the extended mode-coupling analysis will be done. The additionally shown curve calculated after a relaxation of $1.4 \times 10^{7} \mathrm{MCS}$ results from an exemplary run by which we wanted to assess to what extent nonequilibrium effects are still present after $4 \times 10^{6}$ MCS. Figure 2 clearly reveals that a relaxation time of $4 \times 10^{6} \mathrm{MCS}$ does not suffice to eliminate all nonequilibrium effects on the length scale probed by the maximum of the static structure factor. The structural relaxation time of the melt is still underestimated. However, the nonequilibrium effects are no longer as pronounced as for the smallest shown relaxation time, where they blur the two-step decay of $\phi_{q}^{s}(t)$ completely. This two-step decay remains present if the melt is further equilibrated. Therefore we argue that the data to be used in the MCT analysis are sufficiently relaxed to admit a test of the general predictions of the theory, i.e. of the form of the correlator's time and wave vector dependence, of the temperature dependence of the various parameters introduced in the theoretical formulae and of the relationship among these parameters.

\section{Theoretical background}

The previous analysis [20] of the tagged particle correlator (incoherent intermediate scattering function) $\phi_{q}^{s}(t)$ was based on the universal form that $\phi_{q}^{s}(t)$ adopts close to the critical temperature in the $\beta$-relaxation regime $[3,4,5,6,12]$

$$
\phi_{q}^{s}(t)=f_{q}^{s c}+h_{q}^{s} G(t) .
$$

The $\beta$-relaxation regime is defined as the time interval where the tagged particle correlator remains close to the nonergodicity parameter $f_{q}^{s c}$, which implies $\left|h_{q}^{s} G(t)\right| \ll 1$ $[3,4,5,6,12]$. Formula (1) remains valid when the idealized theory is extended by the inclusion of further transport processes $[6,11,12]$. In the $\beta$-relaxation regime these processes, which, for simple liquids, have been identified as hopping processes, can be characterized by a single, wave-vector-independent rate, the hopping parameter $\delta$. In general, $\delta$ is larger than zero. One expects $\delta$ to vary in an Arrhenius-like way [10]

$$
\delta \propto \exp \left[-\frac{A}{\kappa_{T} T}\right]
$$

where $A$ is a system-dependent constant and $\kappa_{T}$ stands for the isothermal compressibility [11]. The hopping parameter depends upon the dynamical parameters of the studied glass former $[4,6,11]$ and is therefore quite different for a system evolving according to a deterministic Newtonian dynamics or to a diffusive Brownian dynamics [6]. This difference in the nature of the underlying dynamics also emerges in the theory via the microscopic time scale $t_{0}$ which characterizes the transient short-time decay of a correlator in a time regime, where mode-coupling effects are not yet dominant [3]. In the idealized theory these mode-coupling effects are solely expressed by the separation parameter $\sigma$ which measures the distance to the critical temperature $T_{c}$, i.e.

$$
\sigma=C\left(T_{c}-T\right)
$$

where the crossover from the glassy $(\sigma>0)$ to the liquid dynamics $(\sigma<0)$ takes place. The existence of this dynamic glass transition is independent of both the microscopic interaction and the microscopic dynamics $[3,4,5,6]$. However, the extent to which this dynamic glass transition can be seen in an experiment or in a simulation depends upon the values of the 
microscopic dynamical parameters and upon the $\delta$-term $\{3,4,6,12]$. Both $\sigma$ and $\delta$ specify the distance from the ideal transition point and are therefore important theoretical control parameters that determine the behaviour of the $\beta$-correlator

$$
G(t)=g\left(t / t_{0}, \sigma, \delta t_{0}\right)
$$

in the extended MCT. In the idealized theory $(\delta=0)$ the $\beta$-correlator is a homogeneous function obeying a one-parameter (i.e. $\sigma$ ) scaling law. Rescaling the time by $1 / y$ and $\sigma$ by $y^{2 a}$ merely changes $G(t)$ by a factor $y^{a}$. The exponent $a$ emerging in this homogeneity property of $G(t)$ is uniquely determined by the equilibrium properties of the glass former and characterizes the relaxation of the fluid particles in their cages. Its value for the polymer model under consideration is known from the previous analysis [20]. In the extended MCT the homogeneity property of the $\beta$-correlator is preserved as a two-parameter (i.e. $\sigma$ and $\delta$ ) scaling law [8]

$$
g\left(t / t_{0}, \sigma, \delta t_{0}\right)=y^{-a} g\left(t / t_{0} y, \sigma y^{2 a}, \delta t_{0} y^{1+2 a}\right)=: y^{-a} g(\hat{t}, \hat{\sigma}, \hat{\delta})
$$

if $\sigma$ and $\delta$ simultaneously change according to

$$
\sigma=\hat{\sigma} / y^{2 a} \quad \delta t_{0}=\hat{\delta} / y^{1+2 a} .
$$

However, this scaling property of the extended theory will normally not be observed in an experiment or simulation, since with the choice $y=|\sigma|^{-1 / 2 a}$ it requires $\delta$ to vary critically, i.e. $\delta t_{0} \propto\left(T_{c}-T\right)^{(1+2 a) / 2 a}$. Due to (2) such a temperature dependence is not expected. Therefore an experimental glass former will not follow one specific scaling line, but move along a path $C: T \rightarrow(\sigma(T), \delta(T))$ which crosses many scaling lines that are labelled by different rescaled parameters $\hat{\delta}=\delta t_{0} / \sigma^{(1+2 a) /(2 a)}$. The path $C$ is fixed by the microscopic details of the given glass former. It cannot be varied and avoids the ideal glass singularity as long as $\delta \neq 0[8,10,12]$.

The previous analysis of the tagged particle correlator in the framework of the idealized MCT [20] exhibited discrepancies between theory and simulation, which could be qualitatively attributed to the neglect of hopping processes. In order to check whether this interpretation may be confirmed quantitatively, the simulation data have to be reanalysed by the extended MCT. This analysis proceeds in the following way. Due to the homogeneity property (5) one may choose $y=|\sigma|^{-1 / 2 a}$, whence

$$
\begin{aligned}
\phi_{q}^{s}(t)=f_{q}^{s c} & +h_{q}^{s} G(t)=f_{q}^{s c}+h_{q}^{s} \sqrt{|\sigma|} g\left(t|\sigma|^{1 / 2 u} / t_{0}, \pm 1, \delta t_{0} /|\sigma|^{(1+2 a) / 2 a}\right) \\
= & f_{q}^{s c}+h_{q}^{s} t_{0}^{a}\left[\frac{1}{t_{\sigma}^{a}} g(\hat{t}, \pm 1, \hat{\delta})\right]
\end{aligned}
$$

with $t_{\sigma}:=t_{0} /|\sigma|^{1 / 2 a}$. A priori, this formula contains five unknown parameters: the nonergodicity parameter $f_{q}^{s c}$, the critical exponent $a$, the total prefactor of the $\beta$-scaling function $h_{q}^{s} t_{0}^{a}$, the time scale of the $\beta$-process $t_{\sigma}$ and the scaled hopping parameter $\hat{\delta}$.

However, we will fix the nonergodicity parameter and the critical exponent at the values obtained in the idealized analysis [20], i.e. at

$$
a=0.239 \quad f_{q}^{s c}=0.80 .
$$

Theoretically, both $a$ and $f_{q}^{s c}$ are unaffected by the inclusion of hopping processes. Therefore it should be possible to determine them reliably by the idealized MCT. Even if hopping processes are important for the relaxation behaviour of the correlator, there is a temperature interval on the liquid side (i.e. $\sigma<0$ ), where the idealized theory is supposed to apply. This temperature interval is limited from below by a temperature $T^{*}$ defined via $\left|\sigma\left(T^{*}\right)\right|=:\left|\sigma_{0}\right|=\left(\delta t_{0}\right)^{2 a /(1+2 a)}$ [see (7)], at which hopping processes start to become 
dominant. From above it is limited by a temperature at which corrections to the leadingorder expressions (1), (4) and (5) can no longer be neglected. In practical applications the lower limit $T^{*}$ is hard to determine unambiguously. Data belonging to this transition region may still be fitted reasonably by the idealized theory if the critical exponent $a$ is allowed to increase with decreasing temperature. Such an observation was made in the mentioned light scattering study of CKN and salol [13]. However, since this kind of force fitting the idealized theory could not compete with an extended MCT analysis when approaching $T_{c}$ more closely, it was suggested in [13] to optimize the idealized fits at short times (i.e. at $t<t_{\sigma}$, where $\delta$-effects are either small or completely negligible [12]) and to accept deviations at long times [13]. Exactly this strategy was pursued in the previous analysis of $\phi_{q}^{s}(t)$ in the framework of the idealized MCT [20] so that the determined value for $a$ should be reliable.

After fixing $a$ and $f_{q}^{s c}$ three parameters $h_{q}^{s} t_{0}^{a}, t_{\sigma}$ and $\hat{\delta}$ remain to be adjusted. This was done by the following two steps. First, the complete $\beta$-scaling function was calculated numerically [12] for a specific value of $\hat{\delta}$ in (7). Then $g(\hat{t}, \pm 1, \hat{\delta})$ was inserted in (7) and the remaining two fit parameters $\left(h_{q}^{s} t_{0}^{a}\right.$ and $\left.t_{\sigma}\right)$ were optimized by inspection. These two steps were iterated until the quality of the fit was satisfactory.

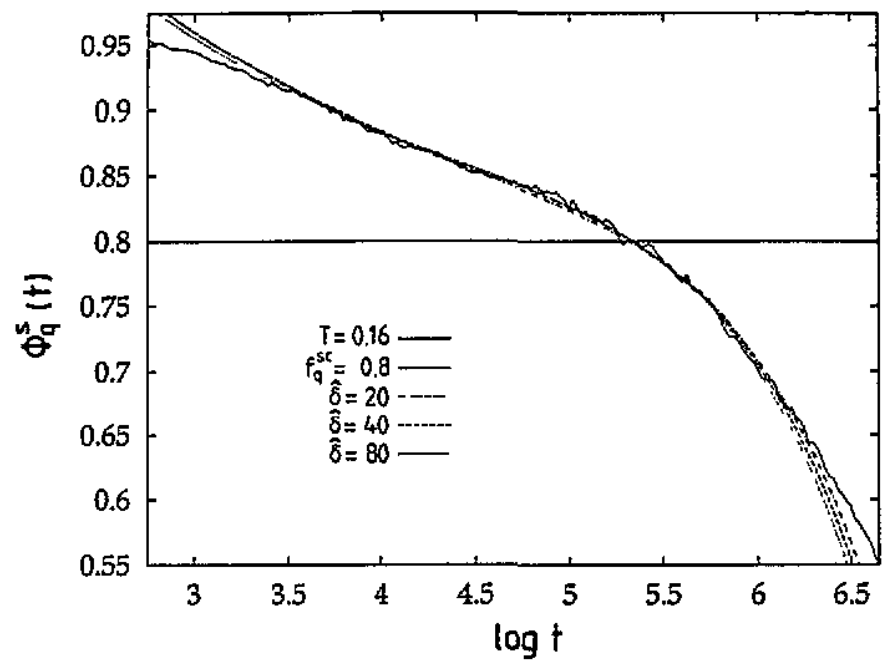

Figure 3. Comparison of fit curves for different $\hat{\delta}$-values at $T=0.16$. The solid curve represents the simulation data, and the horizontal solid line indicates the value of the nonergodicity parameter. The dash-dotted, dashed and dotted curves correspond to the fit by (7) with $\hat{\delta}=20$, $\hat{\delta}=40$ and $\hat{\delta}=80$, respectively. The curves with $\hat{\delta}=20$ and $\hat{\delta}=80$ serve to calculate the errors on the fit results obtained for $\hat{\delta}=40$.

Figure 3 exemplifies this fit procedure for $T=0.16$. The nonergodicity parameter (horizontal solid line) helps to guide the fit because its intersection point with the simulation data (solid curve) defines the time at which $G(t)$ vanishes. This matching of the simulation and theoretical results at $f_{q}^{s c}$ determines $t_{\sigma}$. Finally, one can tune $h_{q}^{s} t_{0}^{a}$ in order to describe the decay of the simulation data as well as possible. In this way the dashed, the dotted and the dash-dotted line were obtained. While the dashed line represents $\hat{\delta}=40$ (the result to be discussed further in the following section), the other two lines correspond to two extreme $\hat{\delta}$-values (i.e. $\hat{\delta}=80$ (dotted line) and $\hat{\delta}=20$ (dash-dotted line)), which still yield fits of similar quality. Increasing or decreasing $\hat{\delta}$ further ruins the fit. Therefore these $\hat{\delta}$-values were used to determine the error of $\hat{\delta}$ at $T=0.16$. In the same way the error of $\hat{\delta}$ was 
obtained at the other temperatures. Since a variation of $\delta$ simultaneously affects all other fit parameters [13], it can be used to estimate the uncertainties on both $\sigma$ and $\delta$.

From these fits it is possible to extract the temperature dependence of the separation parameter $\sigma$ and the hopping parameter $\delta$ by (see (7))

$$
\delta=\hat{\delta} \frac{t_{0}^{2 a}}{t_{\sigma}^{1+2 a}} \quad \sigma=\left[\frac{t_{0}}{t_{\sigma}}\right]^{2 a}
$$

if the change of the microscopic time scale $t_{0}$ with temperature is known additionally. In the previous idealized analysis [20] the temperature dependence of $t_{0}$ was obtained by fitting the initial decay of $\phi_{q}^{*}(t)$ with the short-time expansion of the tagged particle corrclator in the framework of the Rouse model $[33,37]$

$$
\phi_{q}^{s}(t) \approx \exp \left[-q^{2} b^{2} \sqrt{t / t_{0}}\right] \quad \text { for } t_{\text {cross }}<t \ll \tau_{R}
$$

which is valid for times that are larger than a crossover time $t_{\text {cross }}$, below which the microscopic details of the model affect the dynamics of the monomers, and also much smaller than the Rouse time $\tau_{R}$, i.e. the longest relaxation time of a (nonentangled) chain. In $(10) b^{2}$ stands for a mean square bond length which is weakly temperature dependent (i.e. $b^{2}=8.63,8.58$ at $T=0.16,0.21$, respectively) [29]. The result of the fit by (10) is reproduced in some figures of the next section for the sake of comparison with the idealized analysis [20]. Mathematically, the time scale $t_{0}$ enters the mode-coupling theory by matching (7) to the transient microscopics in the vicinity of $T_{c}[3,12]$. It has not been possible to determine the temperature dependence of $t_{0}$ beyond the asymptotic result, $t_{0} \approx t_{0}\left(T_{c}\right)=$ constant, yet. Whereas this asymptotic result appears to hold in simple liquids $[13,14,15,16,38,39]$, the previous analysis of the initial decay of $\phi_{q}^{s}(t)$ by $(10)$ [20] resulted in a strong temperature dependence of the transient microscopic time scale $t_{0}$. This strong temperature dependence can be expected to shift the theoretical matching parameter $t_{0}$. In the following we will therefore assume that the time scale taken from the Rouse analysis determines the temperature dependence of the mode coupling $t_{0}$.

Since the time scale $t_{0}$ enters tho prefactor $h_{q}^{s} t_{0}^{a}$, this prefactor should exhibit the same temperature dependence as $t_{0}$, provided $h_{q}^{s}$ is constant. Asymptotically, this is expected in the vicinity of $T_{c}$, where all quantities that do not vary critically with temperature (i.e. that neither vanish nor diverge in the vicinity of $T_{c}$, such as $f_{q}^{s c}, h_{q}^{s}, a$ and $\delta$ ) may be replaced by their values for $T=T_{c}$ in leading order [3, 12]. In order to test to what extent the studied model reflects this theoretical expectation the temperature dependence of $h_{q}^{s} t_{0}^{\alpha}$ can be compared with that of $t_{0}$ derived by the Rouse model. Yet, it has to be added that a temperature dependence of $t_{0}$ cannot be unambiguously distinguished from a temperature dependence of $h_{q}^{s}$ in our analysis due to the scaling property of (7) [13]; a temperature dependence of the critical amplitude is expected in light scattering experiments [39]. The above reasoning therefore rests upon the polymeric character of the short-time dynamics.

Besides the short-time fit by the Rouse model the figures of the subsequent sections will also reproduce the Kohlrausch function

$$
\phi_{q}^{s}(t)=f_{q}^{s c} \exp \left[-\left(t / \tau_{K}\right)^{\beta_{K}}\right]
$$

of the idealized analysis [20]. This function was used with a temperature-independent exponent $\beta_{K}=0.515$ to describe the final structural decay, i.e. the $\alpha$-relaxation [3], of $\phi_{q}^{s}(t)$. A constant value for $\beta_{K}$ implies the time-temperature superposition principle which is a central result of the idealized MCT for the $\alpha$-process [3]. In the presence of hopping processes it is not completely clear to what extent the time-temperature superposition principle is still relevant for the $\alpha$-relaxation $[8,11,12]$. 


\section{Extended mode-coupling analysis}

This section reports the results of the extended MCT analysis for the incoherent intermediate scattering function $\phi_{q}^{x}(t)$ which was calculated at the maximum of the static structure factor (i.e. at $q=2.92$ [30]) over a time interval of $5 \times 10^{6}-10^{7} \mathrm{MCS}$ in the temperature range $T \in[0.16,0.21]$ [20]. This temperature range was split into two parts by the analysis with the idealized MCT. For $T \geqslant 0.19$ the idealized theory could account for the decay of $\phi_{q}^{s}(t)$ over an intermediate time interval of about three decades. This interval was preceded by one in which the correlator was well described by (10), and succeeded by another where (11) yielded a good fit. For $T<0.19$ the idealized theory extends the initial fit by the Rouse model for about one decade to longer times, but then systematically underestimates the ability of $\phi_{q}^{s}(t)$ to relax. This discrepancy between the idealized theory and the simulation data becomes progressively pronounced with decreasing temperature and may be rationalized by the neglect of hopping processes [20].

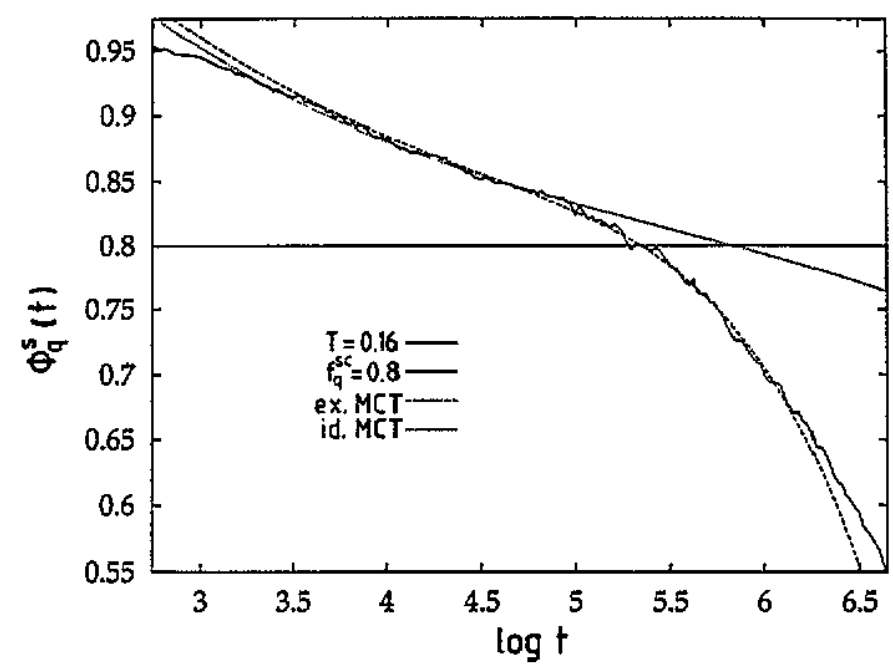

Figure 4. Comparison of the fit by the idealized (dotted curve) and extended (dashed curve) MCT for the simulation data (solid curve) at $T=0.16$. The solid horizontal line is the value of the nonergodicity parameter.

\subsection{Line shape analysis}

Figure 4 compares the idealized fit with that of the extended MCT for the temperature $T=0.16$ at which the deviation between idealized theory and simulation was largest. This comparison shows that the extended analysis increases the interval of the fit for more than one decade to longer times. The extended MCT thus successfully describes the onset of the $\alpha$-relaxation of $\phi_{q}^{s}(t)$, which could not be achieved by the idealized analysis. In order to obtain this improvement at long times it seems that one has to allow the extended fit to become worse than the idealized one at the beginning of the two-step process (i.e. in the time interval between $10^{3}$ and $10^{4} \mathrm{MCS}$ ). However, an extension of the fit to shorter times merely increases the overlap between the MCT and the Rouse model, as can be seen from figure 5. Such an increase is fortuitous and cannot be considered as an argument in favour of the idealized fit. 


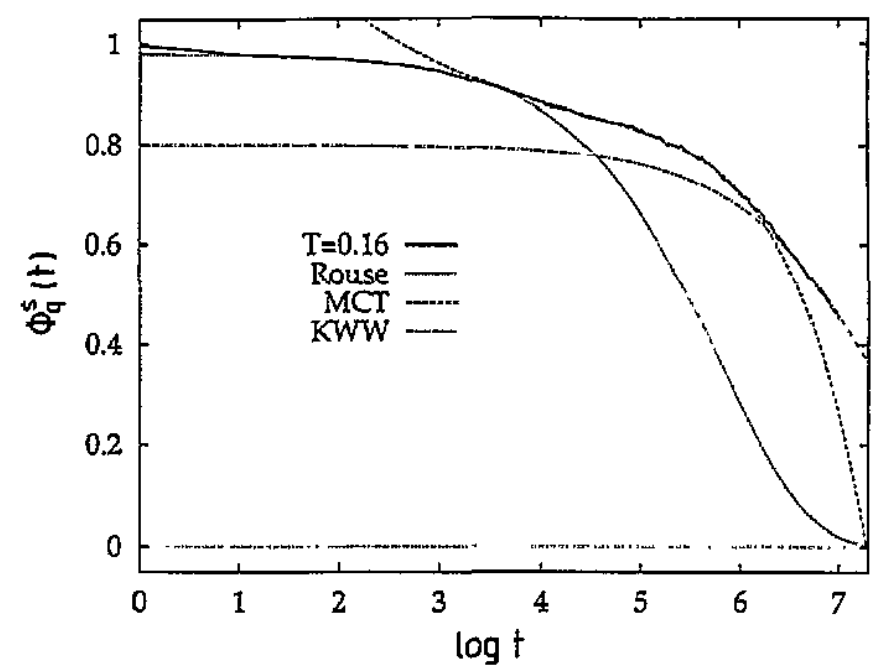

Figure 5. Comparison of the simulation data (solid line) and various fitting formulae at $T=0.16$. The short-time expansion of the Rouse model and the Kohlrausch law (KWW) are represented by a dotted line and by a dash-dotted line, respectively. The dashed line corresponds to the fit by the extended MCr using the data compiled in table 3.

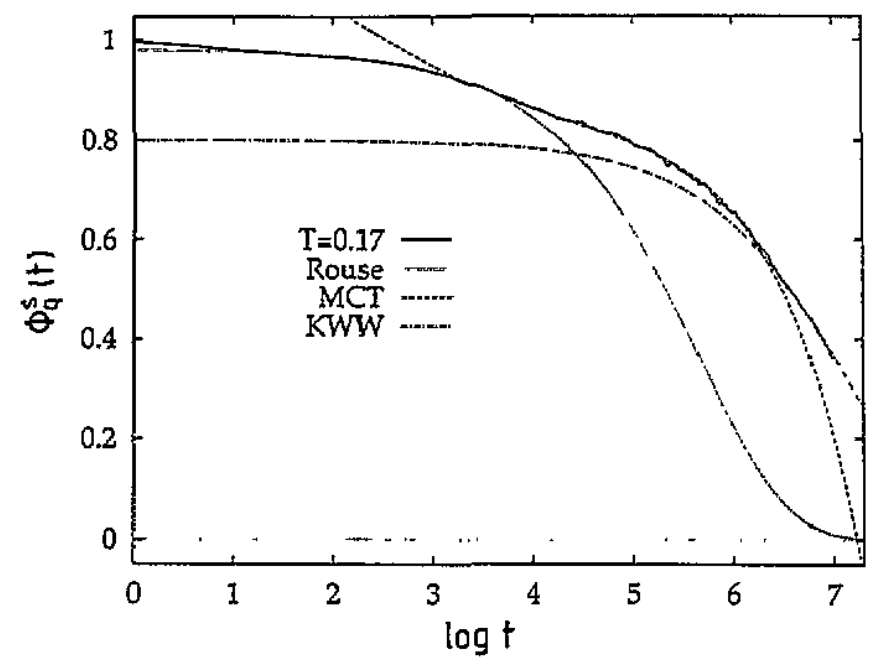

Figure 6. Comparison of the simulation data with the same fitting formulae and the same choice for the types of line as in figure 5 at $T=0.17$.

Figures 5,6 and 7 corresponding to $T=0.16,0.17,0.18$ show that the extended MCT starts to describe the decay of $\phi_{q}^{s}(t)$ just at the edge of the interval where the Rouse model still applies, and then continues the description for about 2.5 decades (i.e. from about $5 \times 10^{3}$ to $10^{6} \mathrm{MCS}$ ) to longer times. When the MCT begins to deviate from the simulation data, the Kohlrausch function continues the fit to arbitrarily long times.

The result of the extended analysis in the low-temperature interval $T \in[0.16,0.18]$ may be summarized in the following way: figures 5 to 7 show that the extended MCT can describe the decay of $\phi_{q}^{s}(t)$ in the $\beta$-relaxation regime and thus resolves the discrepancies found in the previous idealized analysis, which means that in this simulation hopping 


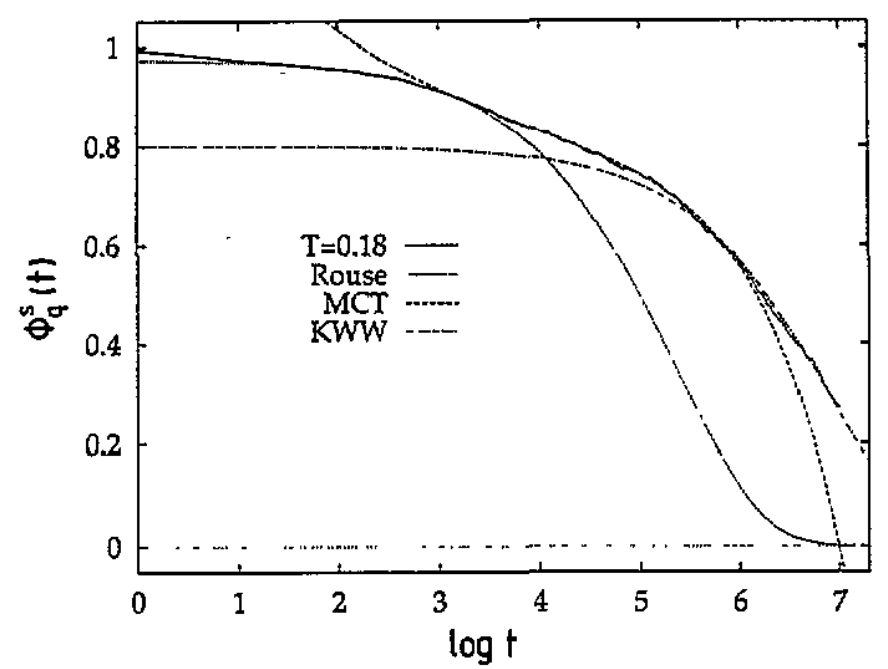

Figure 7. Comparison of the simulation data with the same fitting formulae and the same choice for the types of line as in figure 5 at $T=0.18$.

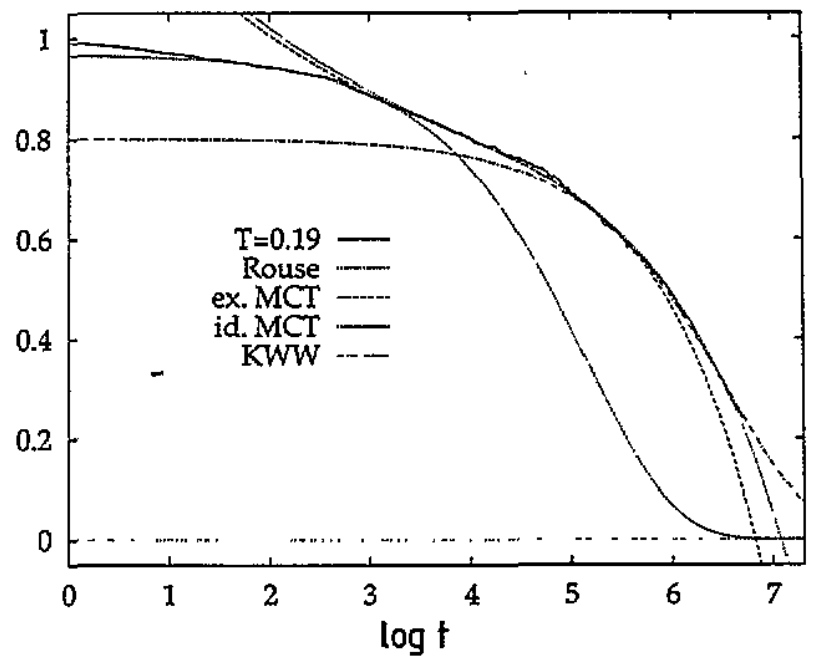

Figure 8. Comparison of the simulation data with the same fitting formulae and the same choice for the types of line as in figure 5 at $T=0.19$. In addition, the fitted curve obtained from the idealized MCT is reproduced from [20] as a dotted line.

processes are important for the relaxation of the tagged particle correlator.

In the high-temperature region $T \in[0.19,0.21] \delta \neq 0$ fits were performed in order to estimate the errors of our analysis in the same way as for low temperatures. The results as well as those for $T \in[0.16,0.18]$ are compiled in the tables $1-3$. By choosing $\delta>0$ one cannot improve the quality of the fit except at $T=0.19$. The reason for this is that $\delta$ increases only weakly at high temperatures (see (2)) in comparison to the increase of $\sigma^{(1+2 a) /(2 x)}$. Therefore $\hat{\delta}$ in (7) becomes negligible for $T \geqslant 0.20$. The temperature $T=0.19$ belongs to the region close to $\left|\sigma_{0}\right|$ (see the discussion following (8)) so that hopping processes contribute noticeably to the relaxation $\phi_{q}^{s}(t)$, although a fit by the idealized theory still yields a reasonable result. Figure 8 compares the idealized and extended analysis at 
$T=0.19$. It shows that the extended fit works better in the time interval which can be covered neither by the Rouse model nor by the Kohlrausch function. Therefore the choice $\delta \neq 0$ is preferable.

Table 1. Fit results to determine the lower bound of the error on the data compiled in table 3.

\begin{tabular}{lllllll}
\hline$T$ & $h_{q}^{s} t_{0}^{g}$ & $t_{0}^{\mathrm{MCT}}$ & $\log t_{\sigma}$ & $\sigma \times 10^{2}$ & $\hat{\delta}$ & $\delta \times 10^{8}$ \\
\hline 0.21 & 0.59 & 4.78 & 4.05 & -2.42 & 0.02 & 4.16 \\
0.20 & 0.66 & 7.64 & 4.42 & -2.04 & 0.04 & 3.10 \\
0.19 & 0.70 & 9.77 & 4.75 & -1.59 & 0.06 & 1.70 \\
0.18 & 0.76 & 13.38 & 5.125 & -1.24 & 0.3 & 2.80 \\
0.17 & 0.88 & 25.45 & 5.72 & -0.87 & 1.5 & 2.48 \\
0.16 & 0.875 & 24.85 & 6.445 & -0.39 & 20 & 2.77 \\
\hline
\end{tabular}

Table 2. Fit results to determine the upper bound of the error on the data compiled in table 3 .

\begin{tabular}{lllllll}
\hline$r$ & $h_{q}^{s} t_{0}^{\prime \prime}$ & $t_{0}^{\text {MCT }}$ & $\log t_{\sigma}$ & $\sigma \times 10^{2}$ & $\hat{\delta}$ & $\delta \times 10^{8}$ \\
\hline 0.21 & 0.555 & 3.93 & 4.01 & -2.26 & 0 & 0 \\
0.20 & 0.63 & 5.0 & 4.38 & -1.94 & 0 & 0 \\
0.19 & 0.67 & 7.64 & 4.78 & -1.41 & 0.2 & 4.69 \\
0.18 & 0.725 & 11.31 & 5.185 & -1.06 & 0.6 & 4.15 \\
0.17 & 0.84 & 20.95 & 5.77 & -0.75 & 2.5 & 3.17 \\
0.16 & 0.84 & 20.95 & 6.75 & -0.25 & 80 & 3.62 \\
\hline
\end{tabular}

Table 3. Values of $h_{q}^{s} t_{0}^{a}, t_{0}^{\mathrm{MCT}}, t_{\sigma}, \sigma, \hat{\delta}$ and $\delta$ corresponding to the results presented in figures 512.

\begin{tabular}{lllllll}
\hline$T$ & $h_{q}^{s} t_{0}^{f f}$ & $t_{0}^{\mathrm{MCT}}$ & $\log t_{\sigma}$ & $\sigma \times 10^{2}$ & $\hat{\delta}$ & $\delta \times 10^{8}$ \\
\hline 0.21 & 0.57 & 4.14 & 4.03 & -2.34 & 0 & 0 \\
0.20 & 0.645 & 6.94 & 4.4 & -1.99 & 0 & 0 \\
0.19 & 0.685 & 8.92 & 4.75 & -1.53 & 0.1 & 2.72 \\
0.18 & 0.745 & 12.68 & 5.15 & -1.16 & 0.4 & 3.29 \\
0.17 & 0.855 & 22.56 & 5.73 & -0.81 & 1.8 & 2.71 \\
0.16 & 0.87 & 24.26 & 6.6 & -0.32 & 40 & 3.23 \\
\hline
\end{tabular}

The results presented so far can be summarized graphically by plotting the $\beta$-scaling function $g(\hat{t}, \pm 1, \hat{\delta})$ versus the reduced time $\hat{t}=t / t_{\sigma}$ for all temperatures of the interval $T \in[0.16,0.21]$. Such a plot is shown in figure 9 . If the decay of $\phi_{q}^{s}(t)$ in the $\beta$-relaxation regime were accurately described by the idealized MCT, one would expect the simulation data to collapse onto the temperature-independent idealized master curve $g(\hat{t}, \pm 1, \hat{\delta}=0)$ for intermediate times and to follow this curve for longer, the smaller the temperature is. In order to exemplify this behaviour, figure 9 shows $g(\hat{t}, \pm 1, \hat{\delta}=0)$ as a dashed line in addition to the scaling curves for finite $\hat{\delta}$ (solid lines). Only $T=0.20,0.21$ satisfy the prediction of the idealized MCT. For these two temperatures no major improvement of the fit quality can be achieved by the inclusion of hopping processes. However, for $T \leqslant 0.19$ deviations from the idealized $\beta$-scaling function become clearly visible. One has to choose $\hat{\delta} \neq 0$ to obtain a good description over a time interval comparable to that of the idealized 


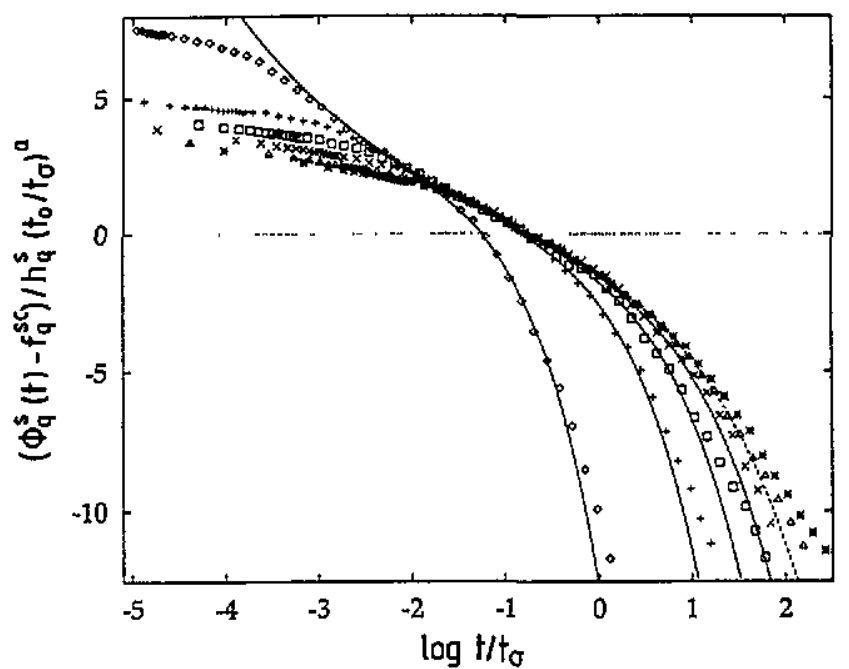

Figure 9. Plot of the $\beta$-scaling function $g(\hat{t}, \pm 1, \hat{\delta})=\left(\phi_{q}^{s}(t)-f_{q}^{s c}\right) / h_{q}^{s}\left(t_{0} / t_{\sigma}\right)^{a t}$ versus the reduced time $\hat{t}=t / t_{\sigma}$. The symbols refer to: $T=0.16(0), T=0.17(+), T=0.18$ (口), $T=0.19(x), T=0.20(\Delta)$ and $T=0.21(*)$. The solid lines correspond to the theoretically calculated scaling functions at the respective temperatures. For $T=0.20,0.21$ the idealized $\beta$-scaling function (i.e. $g(\hat{t}, \pm 1,0))$ is shown as a dashed line.

MCT at $T=0.20,0.21$ (i.e. for two to three decades). The theoretical curves in this figure demonstrate again that the effects of the hopping parameter, which modify the idealized master function, become noticeable at long rescaled times.

\subsection{Hopping parameter and critical temperature}

With the extended analysis of the line shape of $\phi_{g}^{s}(t)$ in the $\beta$-relaxation regime one can derive a more precise estimate of $T_{c}$ and additionally obtain the temperature dependence of the hopping parameter $\delta$ by virtue of (9). These equations need the microscopic time scale $t_{0}$ as input. As mentioned above the temperature dependence of $t_{0}$ can be obtained either from the Rouse fit (denoted by $t_{0}^{\mathrm{RM}}$ [20]) or from the prefactor $h_{q}^{s} t_{0}^{a}$ (denoted by $t_{0}^{\mathrm{MCT}}$ ) if $h_{q}^{s}$ is a constant in the considered temperature range. In order to extract the temperature dependence of $h_{q}^{s}$ figure 10 shows the ratio $h_{q}^{s}\left(t_{0}^{\mathrm{MCT}}\right)^{a} /\left(t_{0}^{\mathrm{RM}}\right)^{a}$ versus temperature. This ratio decreases slightly with falling temperature. However, the change is so weak that it may be approximated by a constant, say by $h_{q}^{s}=0.406$, the average of $h_{q}^{s}\left(t_{0}^{\mathrm{MCT}}\right)^{a} /\left(t_{0}^{\mathrm{RM}}\right)^{a}$ in the interval $T \in[0.17,0.2]$. In the following the $t_{0}^{\mathrm{MCT}}$-values resulting from $h_{q}^{s}=0.406$ will be used instead of $t_{0}^{\mathrm{RM}}$ (tables $1-3$ ). With this choice the subsequent analysis becomes self-consistent in the sense that it only involves fit results of the extended MCT.

With $t_{0}^{\mathrm{MCT}}$ and the other values of tables 1 and 2 the range of $\delta$-values at a given temperature can be calculated, which produces fits of comparable quality. Due to (9) this range has to increase with the distance to the critical temperature, which is clearly visible in figure 11. This figure and table 3 indicate that the hopping rate $\delta$ is (essentially) independent of temperature in this polymeric system. Nevertheless, the effects of hopping which distort the $\beta$-correlator increase when $T$ is decreased. This can be seen from the fits in figures 5-8 and from the increase of the rescaled parameter $\hat{\delta}$ in table 3 .

At first sight, a temperature-independent hopping parameter seems to contradict (2) 


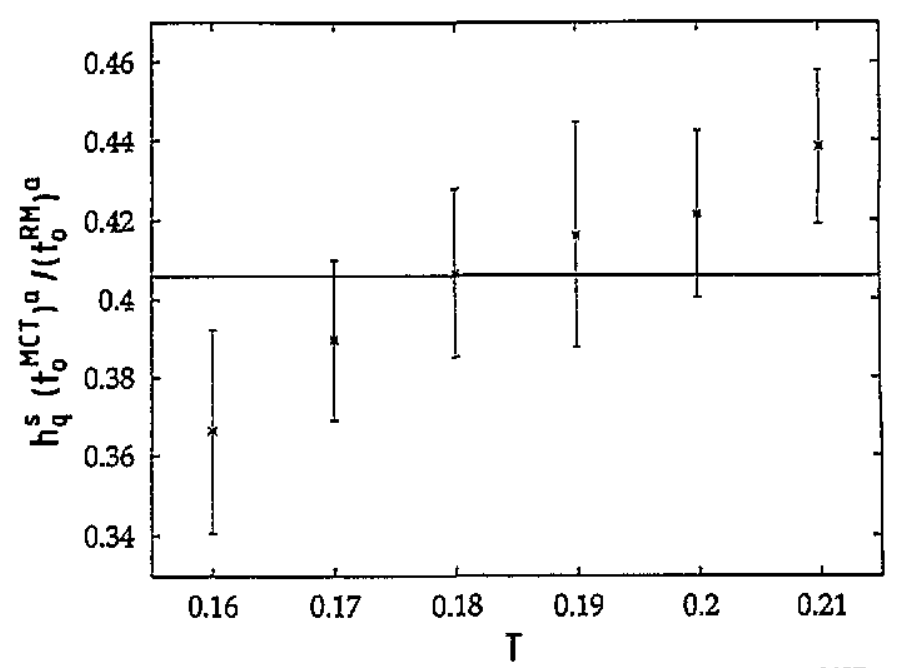

Figure 10. Temperature dependence of $h_{q}^{s}$ resulting from the ratio $h_{q}^{s}\left(t_{0}^{\mathrm{MCT}}\right)^{t t} /\left(t_{0}^{\mathrm{RM}}\right)^{t}$. The data for the microscopic time scale derived by the Rouse model $\left(t_{i}^{\mathrm{RM}}\right)$ are taken from [20]. $h_{q}^{s}=0.406$ (solid horizontal line) was chosen for the subsequent analysis presented in figures 11-13.

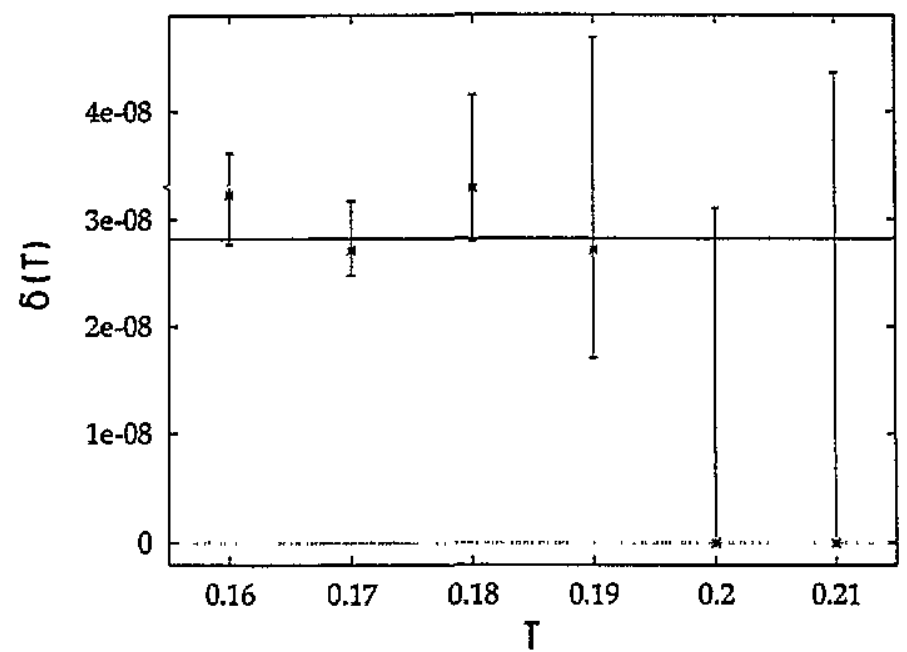

Figure 11. Plot of $\delta$ versus temperature. The stars represent the results from table 3. The errors are calculated from tables 1 and 2. Within the error bars $\delta$ is constant in the considered temperature interval. In addition, a solid horizontal line at $\delta=2.82 \times 10^{-8}$ is shown, which resulted from the least-squares fit presented in figure $\mathbf{1 3}$.

which was experimentally confirmed in the above-mentioned light scattering studies of $\mathrm{CKN}$, salol and PC. However, one has to take into account that the separation parameter $\sigma$ of these studies varied over a large temperature range including also temperatures below $T_{c}$, whereas this simulation is limited to temperatures above $T_{c}$. Figure 12 shows that $\sigma$ varies between $-2.5 \times 10^{-3}$ and $-2.5 \times 10^{-2}$ in this simulation. If one looks at the change of the hopping parameter for comparable $\sigma$-values in the cited light scattering studies one finds that the temperature variation of $\delta$ is rather weak and almost indistinguishable from a constant for some of the materials studied. 


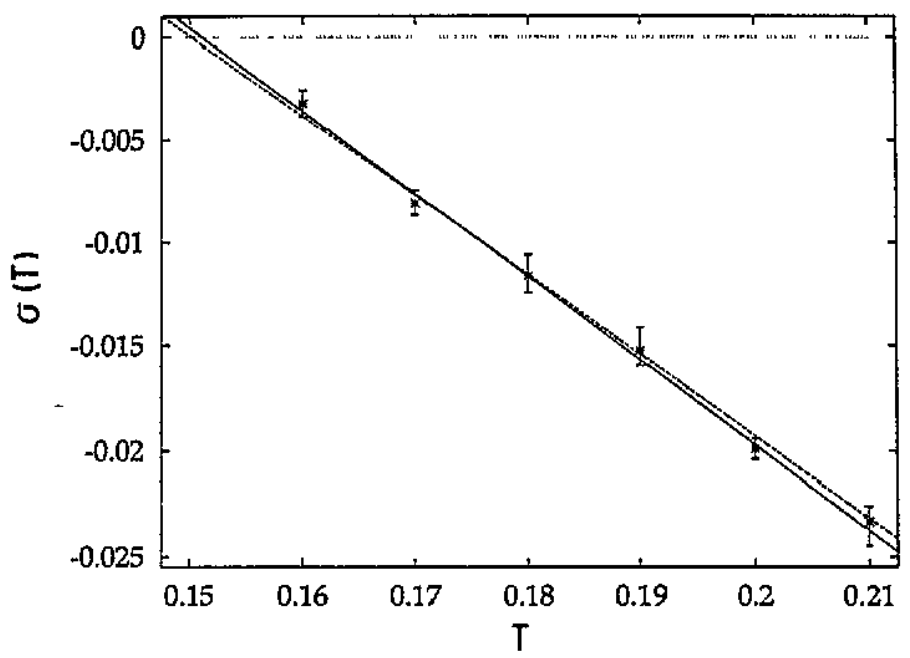

Figure 12. Plot of $\sigma$ versus temperature. The stars represent the results from table 3 . The enrors are calculated from tables 1 and 2 . The solid line is a least-squares fit, yielding a critical temperature of $T_{c}=0.151$. The dashed line resulted from the fit shown in figure 13 , in which $C$ and $T_{c}$ of (3) as well as $\delta$ were kept as temperature-independent constraints.

Another argument supporting the idea of a constant hopping parameter rests upon the temperature dependence of the isothermal compressibility $\kappa_{T}$. This temperature dependence was studied in [36], where it was found that the product $\kappa_{T} T$ is essentially constant in the interval $T \in[0.16,0.21]$. Since this product determines the whole temperature dependence of $\delta$ due to (2), one would not expect the hopping parameter to vary much during the simulation.

The arguments presented can rationalize why thermally activated processes may lead to the temperature independence of $\delta$ observed in this study. However, they do not exclude the possibility that additional polymer-specific microscopic properties determine this parameter. The hopping processes of the MCT for simple liquids result from the approximate treatment of ring collisions between the fluid particles: the particle in the cage kicks one of its neighbours, which in turn kicks one of its neighbours and so on, until this sequence of collisions transfers parts of the original impetus back to the enclosed particle and accelerates it [8]. Whereas this dynamic backflow effect is the important ergodicity restoring microscopic process in simple liquids, one could conjecture that it is, though present, not as important in polymer melts. On the one hand, polymers are heavy objects. In this respect they are comparable to colloid particles for which it has been found experimentally that hopping processes can be completely neglected [38]. On the other hand, the connectedness between the monomers prevents them from moving far away from the backbone of the chain. However, the question then arises of what the microscopic origin of the hopping processes in a polymer melt could be. At the present stage of this work, the answer can only be speculative. It is conceivable that the connectedness between the monomers, which was above made responsible for the suppression of the backflow effect, induces a kind of a slithering motion of the monomers along the backbone of the chain and thus opens a new relaxation channel, not present in simple liquids. Certainly, the sketched picture is very reminiscent of the reptation idea [24]. However, the conjectured slithering motion is not related to entanglements, as the studied chain length $N=10$ is much shorter than the entanglement length [33]. It is connected to the freezing of the interchain contribution 
to the cages. A similar conjecture of anisotropic local motion was also presented in a molecular dynamics study of amorphous polyethylene [26]. Whether such an anisotropy of the monomeric dynamics develops as $T_{c}$ is approached should be detectable by monitoring the motion of the monomers in the internal coordinate system of a chain. Work in this direction is under way.

The temperature independence of the hopping parameter $\delta$ and the strong increase of the microscopic time scale $t_{0}$ determine the range of applicability of the asymptotic $\beta$-scaling law (7). Theoretically the intermediate time window where this asymptotic scaling law holds is expected to grow when $\left(\sigma, \delta t_{0}\right)$ approach the transition point $(0,0)[12]$. However, the matching point of the Rouse and the $\beta$-dynamics moves to longer times with decreasing temperature (see figures 5-8) due to the above-discussed increase of $t_{0}$. At long times the cut-off for the $\beta$-process is set by the $\alpha$-relaxation. For lower temperatures the hopping rate $\delta$ influences the $\alpha$-relaxation time and restricts the $\beta$-relaxation to times shorter than $\sim 0.02 / \delta$ in our case. Since $\delta$ is independent of temperature, the range of validity of the asymptotic scaling law (7) does not increase if temperature is decreased. This temperature independence of $\delta$ and the increase of $t_{0}$ with decreasing temperature also lead to an unexpected path $C$ in the plane of the two relevant control parameters $\left(\sigma, \delta t_{0}\right)$. For $\sigma$ negative and approaching $0, \delta t_{0}$ increases contrary to the expectation (2) [8] and the experimental finding in simple liquids $[13,14]$. The asymptotic scaling law (7) consequently shows no increasing range of validity upon lowering the temperature.

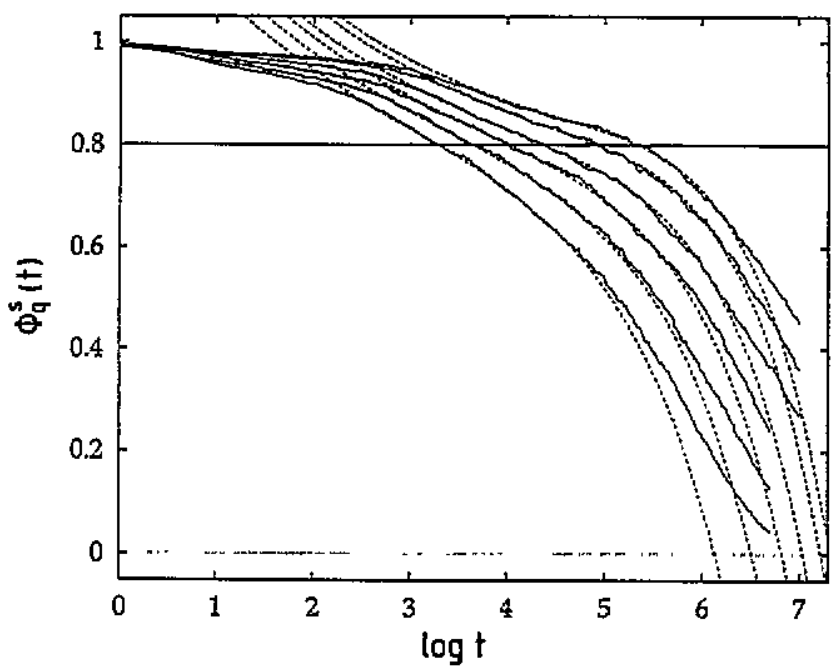

Figure 13. Least-squares fit to the data (solid lines) in the temperature interval $T \in[0.16,0.21]$ (the temperature decreases from left to right in the figure) by optimizing the three (temperatureindependent) parameters, $C$ and $T_{c}$ from (3) as well as $\delta$. The resulting fit parameters are included in figures 11 and 12 . The adjusted time scales $t_{0}^{\text {MCT }}$ are summarized in table 4 . The window $0.6 \leqslant \phi_{q}^{*}(t) \leqslant 0.9$ was chosen for the fit. The horizontal solid line represents the value of the nonergodicity parameter.

An explanation of this finding - also related to the microscopic mechanism determining $\delta$-could be residual aging effects. A glance at figure 2 shows that the $\alpha$-relaxation time increases with the extent of the equilibration of the melt. Due to the above-mentioned connection between the $\alpha$-relaxation time and the cut-off of the $\beta$-process, one can expect the $\beta$-time window to grow when the melt is further equilibrated. This suggests that nonequilibrium effects could also be responsible for the virtual temperature independence 
of $\delta$. This raises the interesting question about the validity of the intermediate $\beta$-scaling law in the presence of nonequilibrium effects and their connection to the parameter $\delta$. The presented analysis of $\phi_{q}^{s}(t)$ would imply that the extended $\beta$-scaling law remains valid and describes the intermediate dynamics close to $T_{c}$, however, with aging effects which strongly affect the hopping parameter $\delta$.

Figure 12 shows the temperature dependence of the separation parameter. As (3) predicts, one finds a linear relationship between $\sigma$ and $T$, yielding $T_{c}=0.151 \pm 0.002$ and $C \approx 0.40$. This value coincides within the error bars with the critical temperature found in the idealized analysis and thus confirms this result.

According to the asymptotic result of the extended MCT the five parameters in (7) are either constant and adopt their values at $T_{c}$ or vary linearly with $T-T_{c}$. In the presented fits the exponent $a$, the nonergodicity parameter $f_{q}^{s c}$ and the critical amplitude $h_{q}^{s}$ were kept constant. The hopping rate $\delta$ turned out to be virtually independent of temperature, and $\sigma$ showed a linear variation with $T-T_{c}$ (see figures 11 and 12). Guided by these results we additionally performed a least-squares fit to the data, in which we tried to find temperatureindependent values for the three parameters $C, T_{c}$ (see (3)) and $\delta$. During the fit only the time scale $t_{0}$ had to be adjusted at each temperature due to the temperature dependence of the Rouse short-time dynamics. The result of this fit is shown in figure 13 , and the parameter values obtained $\left(\delta=2.82 \times 10^{-8}, C=0.387\right.$ and $\left.T_{c}=0.151\right)$ are included in figures 11 and 12. In addition, table 4 compiles the resulting $t_{0}^{\mathrm{MCT}}$-values. The quality of these least-squares fits is comparable to that of the fits presented in figures 5-8, and the determined parameters nicely fall within the previously obtained enror bars (see figures 11 and 12 , for instance).

Table 4. Values of $t_{i}^{\mathrm{MCT}}$ used in the fit with $a, \delta, f_{q}^{s c}$ and $h_{q}^{s}$ independent of temperature and the linear temperature dependence in (3) for $\sigma$; the fit is shown in figure 13.

\begin{tabular}{lr}
\hline$T$ & \multicolumn{1}{c}{$t_{0}^{\mathrm{MCT}}$} \\
\hline 0.21 & 4.00 \\
0.20 & 6.29 \\
0.19 & 9.49 \\
0.18 & 12.40 \\
0.17 & 21.61 \\
0.16 & 26.87 \\
\hline
\end{tabular}

However, it has to be pointed out that the least-squares fitting procedure still contains one aspect where physical reasoning is needed in order to obtain meaningful results. The results are quite sensitive to the choice of the dynamical window in which the fit is optimized. The presented fit was performed to the correlators within the range $0.6 \leqslant \phi_{q}^{s}(t) \leqslant 0.9$. Shrinking the window around the nonergodicity parameter down to $0.7 \leqslant \phi_{q}^{s}(t) \leqslant 0.85$ improves the fit in this range somewhat, but the fit parameters are strongly shifted: $T_{c}$ moves to $T_{c}=$ 0.154 out of the above-given error bar, $\delta$ becomes $\delta=3.5 \times 10^{-8}$ and $C=0.42$. Moreover, this fit can be ruled out by arguments relying on the way corrections to the asymptotic law are expected to behave $[3,6,13]$. At the beginning of the $\beta$-relaxation regime the fit falls below the simulation data at the temperature $T=0.17$, whereas it lies above the data for the other temperatures. Mathematically, this may be interpreted as a change of sign in the shorttime corrections of the $\beta$-master function. This change of sign indicates that the corrections do not vanish due to asymptotic scaling but due to some accidental cancellation effect. A fortuitous cancellation of the corrections to the $\beta$-scaling law requires that the second- 
and third-order corrections are of equal size, which makes doubtful the applicability of the first-order result (7). This shows that the noise of the data may lead to qualitatively wrong least-squares fits, if the fit interval chosen is too small. On the other hand, if the interval is chosen too large, e.g. from $0.5 \leqslant \phi_{q}^{s}(t) \leqslant 0.95$, the numerical procedure interpolates data inside and outside of the actual range of validity of (7). As in the first case a non-monotonic temperature dependence of the corrections is observed and generally the fits worsen also close to the nonergodicity parameter.

\section{Summary}

This paper presents an analysis of the incoherent intermediate scattering function $\phi_{q}^{s}(t)$ in the framework of extended mode-coupling theory. The major results of this application can be summarized as follows. (1) The predicted two-step relaxation behaviour of the extended MCT (beyond the microscopic transients) is observed in the simulation data of the supercooled polymer melt. (2) The extended $\beta$-theory describes the decay of $\phi_{q}^{s}(t)$ over about 2.5 decades in the temperature range $T \in[0.16,0.19]$. Especially for $T<0.19$ the analysis extends the fit by more than one decade to longer times and thus removes the found discrepancies between the simulation data and the idealized analysis in the $\beta$ relaxation regime. (3) During the fit it is possible to keep all quantities that do not vary critically with temperature (i.e. $f_{q}^{s c}, a, h_{q}^{y}$ and $\delta$ ) independent of temperature. The whole temperature dependence is carried by the $\beta$-time scale $t_{\sigma}$. This temperature dependence of $t_{\sigma}$ results from that of the scale $t_{0}$, which describes the matching to the short-time transient, and from the MCT separation parameter $\sigma$. From $\sigma$ one obtains a critical temperature of $T_{c} \approx 0.151$. This value coincides with the idealized result within the error bars. (4) The results of the extended analysis also corroborate the conclusion of the previous paper [20]: the idealized MCT is an useful simplification of the extended theory, provided one applies it while keeping hopping effects in mind. (5) The long-time dynamics of the $\alpha$-relaxation can be fitted by a Kohlrausch function with a temperature-independent exponent. This implies the time-temperature superposition principle.

In summary, the presented analysis shows that $\mathrm{MCT}$, a theory which has been developed for simple liquids, is able to describe quantitatively the relaxation of the considered supercooled polymer melt in the $\beta$-relaxation regime, provided hopping processes are taken into account. The predicted universal dynamical scaling law was monitored for different temperatures and over about three decades in time. How the processes called 'hopping' in this study can be understood in terms of the microscopic properties of the model studicd is still an open question. We speculated that they might be related to the interplay between cage effect and chain connectivity, which could lead to a more or less slithering, reptationlike motion of the monomers (though the studied chains are too short to be entangled). Whether this is true represents an interesting problem for future work. It is also possible that the temperature independence of $\delta$ results from nonequilibrium effects due to insufficient equilibration. In order to answer this question all aging effects have to be eliminated first. This has been achieved recently by using a different dynamical algorithm than the one used here [40]. Therefore we plan to repeat the extended MCT analysis for the fully equilibrated polymer melt, paying then also particular attention to the $q$-dependence of $\phi_{q}^{s}(t)$. Besides this one could make a systematic study of the lifetime of the cages, since the identity and the motion of the neighbours to a given monomers can also be monitored. In this way it might be possible to understand how the fractal decay laws of the MCT are created by the underlying static and dynamic properties of the model. 


\section{Acknowledgments}

We are indebted to Professor K Binder, Professor W Götze, Dr W Kob and Dr W Paul for many helpful discussions and a careful reading of the manuscript. JB is very grateful to the Höchstleistungsrechenzentrum (HLRZ) at Jülich for a generous grant of computer time on the Cray YMP and to the Bundesministerium für Forschung und Technologie (BMFT) for support of this work under grant number 03M4076A3. MF acknowledges financial support from the Deutsche Forschungsgemeinschaft under contract Go 154/8-1.

\section{References}

[1] Jäckle J 1986 Rep. Prog. Phys. 49171

[2] Zallen R 1983 The Physics of Amorphous Solids (New York: Wiley)

[3] Götze W 1990 Liquids, Freezing and the Glass Trunsition ed J P Hansen, D Levesque and J Zinn-Justin (Amsterdam: North-Holland)

[4] Götze W and Sjögren L 1992 Rep. Prog. Phys. 5524 !

[5] Schilling R 1994 Disorder Effects on Relaxational Processes ed R Richert and A Blumen (Heidelberg: Springer)

[6] Götze W 1993 Nato ASI Series C: Mathematical and Physical Sciences No 415, ed T Riste and D Sherrington (Dordrecht: Kluwer)

[7] Das S P and Mazenko G F 1986 Phys. Rev. A 342265

[8] Götze W and Sjögren L 1987 Z. Phys. B 65415

[9] Goldstein M 1969 J. Chem. Phys. 513728

[10] Götze W and Sjögren L, 1988 J. Phys. C: Solid State Phys. 213407

[11] Sjögren L 1990 Z. Phys. B 795

[12] Fuchs M, Götze W, Hildebrand S and Latz A 1992 J. Phys.: Condens. Matter 47709

[13] Cummins H Z, Du W M, Fuchs M, Gotze W, Hildebrand S, Latz A, Li G and Tao N J 1993 Phys. Rev. E 474223

[14] Du W M, Li G. Cummins H Z, Fuchs M, Toulouse J and Knauss L A 1993 Phys. Rev. E 492192

[15] Li G. Du W M, Chen X K. and Cummins H Z and Tao N J 1992 Phys. Rev. A 453867

[16] Li G, Du W M, Sakai A, Cummins H Z and Tao N J 1992 Phys. Rev. A 463343

[17] Zeng X C, Kivelson D and Tarjus G 1994 Phys. Rev. E 501711

[18] Dixon P K, Menon N and Nagel S R 1994 Phys. Rev. E 501717

[19] Cummins $\mathrm{H} \mathrm{Z}$ and Li G 1994 Phys. Rev. E 501720

[20] Baschnagel J 1994 Phys. Rev. B 49 135

[21] Wittmann H-P and Kremer K 1992 Comput. Phys. Commun 71 343; 1990 Comput. Phys. Commun. 61309 Deutsch H-P and Binder K 1991 J. Chem. Phys. 942294

Carmesin I and Kremer K. 1988 Macromolecules 212819

[22] Kremer K and Binder K 1988 Comput. Phys. Rep. 7259

[23] Baschnagel J, Qin K, Paul W and Binder K 1992 Macromolecules 253117

Baschnagel J, Binder K, Paul W, Laso M, Suter U W, Batoulis I, Jilge W and Bürger T $1991 \mathrm{~J}$. Chem. Phys. 956014

[24] Doi M and Edwards S F 1986 Theory of Polymer Dynamics (Oxford: Clarendon)

[25] Wool R P 1993 Macromolecules 261564

[26] Roe R J 1994 J. Chem. Phys. 1001610

Roe R J, Rigby D. Furuya H and Takeuchi H 1992 Comput. Polym. Sci. 232

Rigby D and Roe R J 1990 Macromolecules 23 5312; 1989 Macromolecules 22 2259; 1988 J. Chem. Phys. 89 5280; 1987 J. Chem. Phys. 877285

[27] Clarke J H R and Brown D 1989 Mol. Simul. 3 27; 1986 Mol. Phys. 58815

[28] Clarke J H R 1995 Monte Carlo and Molecular Dynamics, Simuletions in Polymer Science ed K Binder (New York: Oxford University Press) at press

[29] Baschnagel J, Binder K and Wittmann H-P 1993 J. Phys.: Condens. Matter 51597

[30] Temperature is measured in units of $\epsilon / k_{\mathrm{B}}$, where $\epsilon$ is the parameter of the bond vector energy (see text and [29] for further explanations). All length and time scales are measured in units of lattice constants and of Monte Carlo steps, respectively.

[31] Flory P J 1969 Statistical Mechanics of Chain Molecules (New York: Wiley) 
[32] Rosenke K, Sillescu $H$ and Spiess H W 1980 Polymer 21757

[33] Paul W, Binder K, Heermann D W and Kremer K 1991 J. Physique II 137

[34] Paul W 1992 AIP Conf. Proc. 256 I45

[35] Paul W and Pistoor N 1994 Macromolecules 271249

Paul W 1994 J. Non-Cryst. Solids 172-174 682

[36] Baschnagel J and Binder K 1994 Physica A 20447

[37] Baumgärtner A and Binder K 1981 J. Chem. Phys. 752994

[38] van Megen W and Underwood S M 1993 Phys. Rev. Lett 70 2766; 1993 Phys. Rev. E 47248

[39] Fuchs M, Cummins H Z, Du W, Götze W, Latz A, Li G and Tao N J 1995 Phil. Mag. B 71771

[40] Wolfgardt M, Baschnagel J and Binder K $1995 \mathrm{~J}$. Physique at press 